\title{
Place Made People
}

\section{Mitchell Ball}

The dim little venue, dull smoke, big rock and roll—talking small, can't communicate.

Nervousness overcomes words. No need to dwell. Shaky feeling, everyone's looking.

Worried sweating churned shit. Checking fat body tension and fudging guitar.

Whoops.

Need a drink.

Adrenaline turns over. Detached fingertips separating show lights encapsulating-glare, reality.

Hot cigarettes makes throat choke, a glimmer sometimes beautiful.

Then applause.

Distracted I can't talk drunk fuck around. Pretending smile.

Talk back thoughts, compliments.

Oh fuck up. Need cigarette, drink. Need home.

Noise stops. Beer light descends, taking grounded acid love. Calm lounge music feeds ears.

Pass incense joint. Chainsmoke society. Moments swinging beyond understanding,

Surviving fascinated, hidden story explicit.

Recognise conscious mistakes, express-regret rolls out between unsure upbringing.

Time's shadow revealed ... 
Everything is words

I'm reading the world.

I feel with articulate hands. Tiger embraces, mobile kisses and ether undercurrents play the right note.

Interested eyes must see high, longer . . Stop world, need talk . . . Need one more toke.

Next day — voice chokes, loathing senses wake.

Compose for the first coffee and rock and roll smoke

Expression flees

Beyond grasp. 